\title{
SECTIONS OF CONTINUOUS COLLECTIONS
}

\author{
J. H. ROBERTS AND PAUL CIVIN
}

In the present note we establish the following

Theorem. Suppose $G$ is a continuous collection ${ }^{1}$ of closed and compact sets filling a separable metric space $X$. Suppose further that the space $G$, considered as a decomposition space, has dimension at most $n$. Then there is a closed subset $K$ of $X$, such that for each $g \in G$, the set $g \cdot K$ is nonvacuous and consists of at most $(n+1)$ points.

We call such a point set $K$ an $(n+1)$-section of the collection $G$. Thus a 1 -section of $G$ is a true section. G. T. Whyburn ${ }^{2}$ has shown that if the elements of $G$ are 0 -dimensional and $G$ is a dendrite, then $G$ admits a true section. The present result gives only a 2 -section, but there is no hypothesis on the dimension of the elements of $G$. For $n=1$, it is known that in general $G$ does not admit a true section. For $n>1$ it is not known whether the present result gives the best possible constant.

We first establish the theorem in the 0-dimensional case.

Lemma. Suppose $G$ is 0-dimensional, and $\epsilon$ is a given positive number. Suppose $W$ is an open set in $X$ such that $W \cdot g \neq 0$ for each $g \in G$. Then there is an open set $E$ in $X$ such that $\bar{E} \subset W, E \cdot g \neq 0$ for every $g \in G$, and the diameter of $E \cdot g<\epsilon$ for each $g \in G$.

Let $f(x)$ be a homeomorphism of $M$, a subset of the Cantor set, into $G .^{3}$ In the product space $M \times X$, consider the set $A$ of points $(x, y)$ with $x \in M$ and $y \in f(x)$. For $x \in X$ there is a unique $y=y(x)$ in $M$ such that $x \in f(y)$. The function $t(x)=(y(x), x)$ is a homeomorphism of $X$ into $A$.

In the space $A$, the open set $t(x)$ and the continuous collection $H$ of elements $t(g)$ for $g \in G$ satisfy the properties of $W$ and $G$ stated in the hypothesis of the lemma. Furthermore, the diameter of a set $Z$ in $A$ is not smaller than the diameter of $t^{-1}(Z)$. Hence all we need show is that there exists an open set $E$ satisfying the theorem relative to the open set $t(W)=U$ and the continuous collection $H$.

Presented to the Society, April 3, 1942; received by the editors April 30, 1942.

${ }^{1} A$ continuous collection filling a space $X$, is a collection $G$ of sets $g$ such that:

(1) If $x \in X$, then $x \in g$ for exactly one $g$. (2) If $x \in g, x_{n} \in g_{n}$ and $x_{n} \rightarrow x$, then $\lim g_{n}=g$.

${ }^{2} A$ theorem on interior transformations, Bull. Amer. Math. Soc. vol. 44 (1938) pp. 414-416.

${ }^{3}$ P. Urysohn, Sur les multiplicités Cantoriennes, Fund, Math, vol, 7 (1926) p. 77. 
For each $p \in U$ there is an open set $U_{p}$ such that (1) $U_{p} \supset p$, (2) $\bar{U}_{p} \subset U$, (3) the diameter of $U_{p}<\epsilon$, and (4) the projection $V_{p}$ of $U_{p}$ upon $M$ is both open and closed. The collection $\left\{V_{p}\right\}$ is an open covering of $M$ and therefore there is a countable subcollection $\left\{V_{p_{i}}\right\}$ covering $M$. The collection of sets $\left\{W_{i}\right\}$ where $W_{i}$ is defined by the relations

$$
W_{1}=V_{p_{1}}, \quad W_{i}=V_{p_{i}}-\sum_{j=1}^{i-1} V_{p_{j}}
$$

is a covering of $M$ by mutually exclusive open sets. Let $Y_{i}$ denote the open subset of $U_{p_{i}}$ whose projection is $W_{i}$ and let $E=\sum_{i=1}^{\infty} Y_{i}$. The open set $E$ has the required properties relative to the space $A$, the open set $U$, and the continuous collection $H$.

In order to prove that $\bar{E} \subset U$ it is sufficient to show that $\bar{E}=\sum_{i=1}^{\infty} \bar{Y}_{i}$. Suppose $p \in \bar{E}$ and $p \in \sum_{i=1}^{\infty} \bar{Y}_{i}$. Then there is a sequence $p_{n} \rightarrow p$ and $p_{n} \in Y_{i_{n}}$. Suppose $p \in g$, and $\pi(g) \subset W_{j}$, where $\pi$ denotes the projection of $A$ on $M$. Since $p_{n} \rightarrow p, \pi\left(p_{n}\right) \rightarrow \pi(p)$. But $\pi\left(p_{n}\right) \nsubseteq W_{j}$ for more than a finite number of $n$. This contradicts the fact that $W_{j}$ is open.

Now $E$ intersects each $g$ since the sequence $\left\{W_{i}\right\}$ is a covering of $M$. Also, since the sets $W_{i}$ are mutually exclusive, if $Y_{i} \cdot g \neq 0$ then $Y_{j} \cdot g=0$ for $i \neq j$. Then, as $Y_{i} \subset U_{p_{i}}$ and the diameter of $U_{p_{i}}<\epsilon$, the diameter of $E \cdot g<\epsilon$ for every $g \in H$. This proves the lemma.

The theorem for the 0 -dimensional case follows by considering a sequence of positive numbers $\left\{\boldsymbol{\epsilon}_{n}\right\}$ with $\epsilon_{n} \rightarrow 0$ as $n \rightarrow \infty$ and a sequence of open sets $\left\{E_{n}\right\}$ such that $\bar{E}_{n+1} \subset E_{n}, E_{n} \cdot g \neq 0$ for $g \in G$, and the diameter of $E_{n} \cdot g<\epsilon_{n}$, for $g \in G$. The common part $K$ of the sets $E_{n}$ is closed. For $g \in G$, the set $K \cdot g$ consists of exactly one point, since $g$ is compact and $\epsilon_{n} \rightarrow 0$.

The theorem for the $n$-dimensional case follows by considering an at most $(n+1)$-to-one closed mapping $f(x)$ of a subset $M$ of the Cantor set ${ }^{5}$ into $G$. In the product space $M \times X$ consider the set $A$ of points $(y, x)$ with $y \in M$ and $x \in f(y)$. The sets $(y, x)$ for $y$ fixed and $x \in f(y)$ form a 0 -dimensional continuous collection $H$ which fills $A$. The mapping $t(y, x)=x$ is a closed, at most $(n+1)$-to-one mapping of $A$ into $X$. By the theorem for the 0 -dimensional case, there is a true section $K$ of the collection $H$ in the space $A$. The set $t(K)$ gives the required $(n+1)$-section of the continuous collection $G$.

DUKe UnIVERsity

${ }^{4}$ That is, $V_{p}$ is the set of $x \in M$ such that $(x, y) \in U_{p}$.

${ }^{5}$ See J. H. Roberts, $A$ theorem on dimension, Duke Math. J. vol. 8 (1941) p. 572 , Theorem 9.1. The mapping $\phi_{n}$ as actually defined is a closed mapping, although this result is not specifically stated in the theorem. 